\title{
FREQUENCY MODULATION BASED ANGULAR RATE SENSOR
}

\author{
Sergei A. Zotov, Igor P. Prikhodko, Alexander A. Trusov, and Andrei M. Shkel \\ MicroSystems Laboratory, University of California, Irvine, CA, USA
}

\begin{abstract}
We report, for the first time, a quasi-digital angular rate sensor based on mechanical frequency modulation (FM) of the input rotation rate. The new approach relies on tracking of the resonant frequencies of two ultra-high $Q$-factor mechanical modes of vibration in a MEMS vibratory gyroscope to produce a frequency based measurement of the input angular rate. Rate table characterization of the new FM angular rate sensor with quality factors of 1 million revealed a linear dynamic range of $2000 \mathrm{deg} / \mathrm{s}$ limited by the setup, with a fundamental performance limit in excess of $72,000 \mathrm{deg} / \mathrm{s}$. Temperature characterization of the FM rate sensor exhibited less than $0.2 \%$ variation of the angular rate response between $25^{\circ} \mathrm{C}$ and $70{ }^{\circ} \mathrm{C}$ environments, enabled by the self-calibrating differential frequency detection.
\end{abstract}

\section{INTRODUCTION}

Maximization of the mechanical quality factors $(Q)$ is key to improving the performance of micromachined vibratory gyroscopes $[1,2]$. Conventional vibratory rate gyroscopes are operated as analogue Amplitude Modulation (AM) systems, where the mechanical sense-mode response is excited by the input angular rate amplitude-modulated by the drive-mode velocity due to the Coriolis effect. Mode matching of conventional high- $Q$ angular rate gyroscopes increases the signal-to-noise-ratio at the tradeoff of linear input range and measurement bandwidth $(10 \mathrm{deg} / \mathrm{s}$ range, sub-Hz bandwidth typical for $Q$ on the order of $10^{5}$ and above). These constraints stem from a fundamental $Q$ versus bandwidth tradeoff and dynamic range limitations of analog $\mathrm{AM}$ systems. The AM based angular rate sensor operation is also extremely sensitive to the value of the sense-mode $Q$ factor, resulting in significant scale factor drifts over practical variations in the ambient temperature and pressure.

In contrast, an angular rate sensor with intrinsic Frequency Modulation (FM) operation could eliminate the gain-bandwidth tradeoff of conventional AM gyroscopes and enable signal-to-noise ratio improvements by taking advantage of ultra-high $Q$ mechanical sensor elements without limiting the measurement bandwidth and range [3]. At the same time, FM sensor architectures are known to provide inherent robustness against mechanical and electromagnetic interferences [4,5].

This paper reports, for the first time, a novel operating principle for a quasi-digital angular rate sensor based on mechanical frequency modulation of the input rotation rate, Figure 1. This sensor architecture is enabled by a unique combination of a symmetric, ultra-high $Q$, silicon micromachined Quadruple Mass Gyroscope (QMG) and a new quasi-digital signal processing scheme which takes advantage of a previously ignored mechanical FM effect of vibratory gyroscope dynamics, Figure 2.

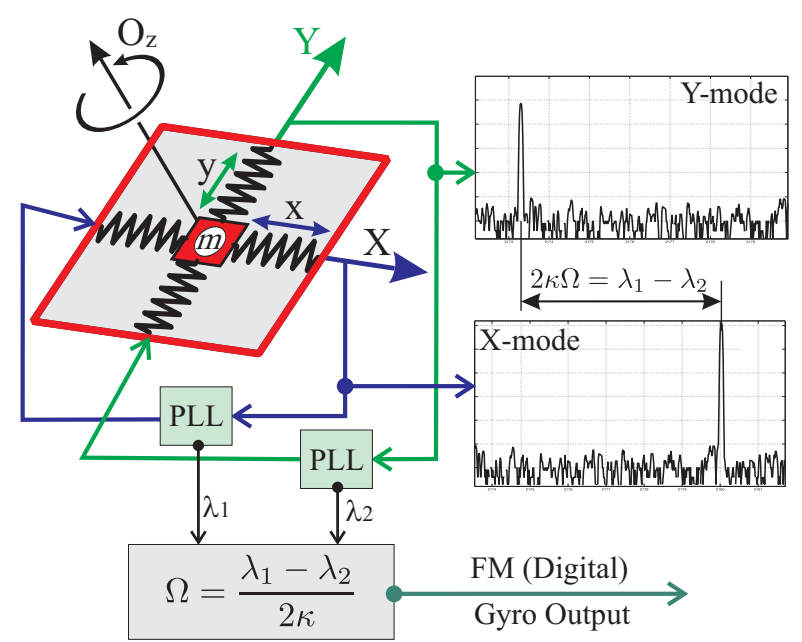

Figure 1: Schematic of the quasi-digital gyroscope based on mechanical Frequency Modulation of the input rate.

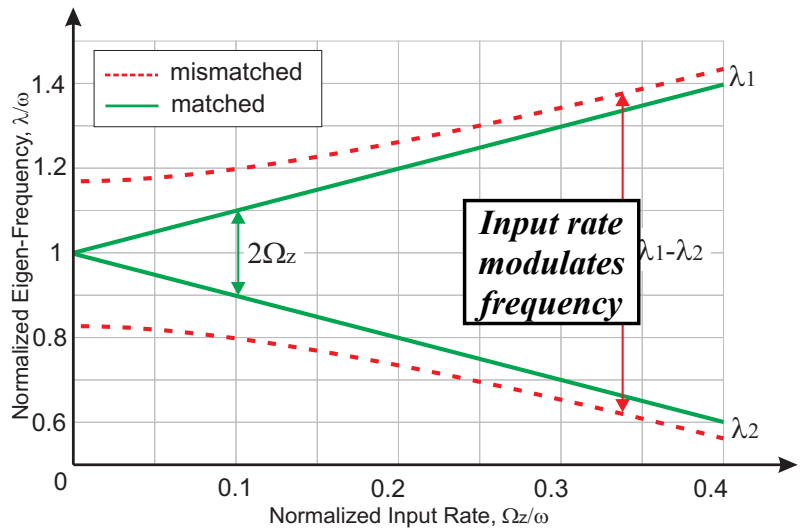

Figure 2: Frequency Modulation of the gyroscope's modal frequencies $\lambda_{i}\left(\Omega_{z}\right)$ by the input angular rate $\Omega_{z}$, simulation.

\section{FREQUENCY BASED RATE DETECTION}

In this section, we analyze the often ignored mechanical frequency modulation effect in vibratory gyroscopes and formulate a new FM based operating principle.

\section{High-Q Gyroscope Dynamics}

Figure 1 shows a conceptual diagram of a $z$-axis vibratory gyroscope considered as a proof mass $m$ suspended in the $x-y$ plane of vibration and sensitive to the $z$-axis rate of rotation $\Omega_{z}$. The free vibrations dynamics of the system in presence of $z$-axis rotation and assuming negligible damping (i.e., ultra-high $Q$ ) is governed by:

$$
\left\{\begin{array}{l}
\ddot{x}+\left((\omega+\Delta \omega / 2)^{2}-\Omega_{z}^{2}\right) x-2 \Omega_{z} \dot{y}=0, \\
\ddot{y}+\left((\omega-\Delta \omega / 2)^{2}-\Omega_{z}^{2}\right) y+2 \Omega_{z} \dot{x}=0,
\end{array}\right.
$$




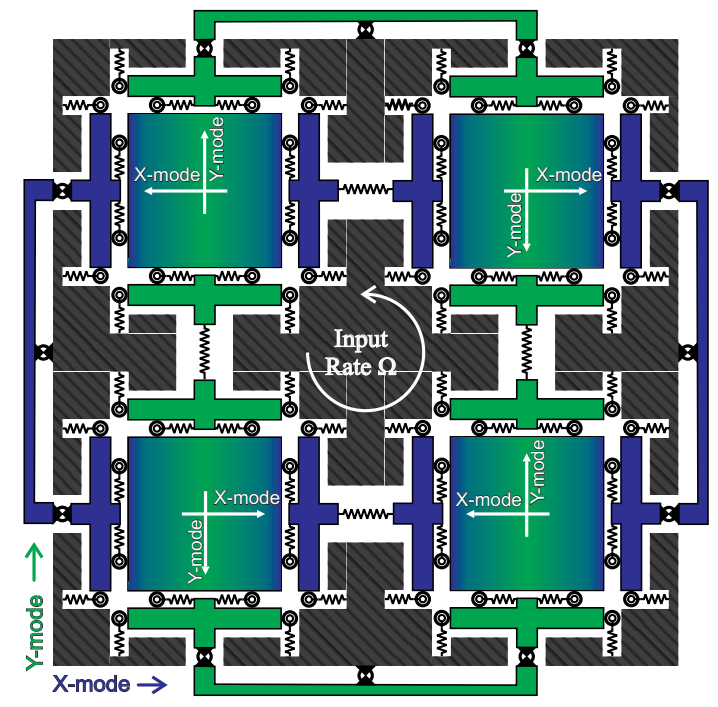

Figure 3: Structural design of the symmetric, dynamically balanced Quadruple Mass Gyroscope ( $Q M G$ ) element.

where $(\omega \pm \Delta \omega / 2)$ are the zero-rate modal frequencies for the $x$ - and $y$-axis mechanical modes, respectively. In this analysis, we deliberately include the often ignored effect of the reverse Coriolis coupling of energy from the $y$-mode back to the $x$-mode, as well as the centrifugal forces.

\section{Rate-Dependent Modal Frequency Splitting}

In presence of the angular rate $\Omega_{z}$, the two modal frequencies (eigen-frequencies) for the gyroscope can be derived as:

$$
\left\{\begin{array}{l}
\lambda_{1}=\sqrt{\Omega_{z}^{2}+\omega^{2}+\Delta \omega^{2} / 4+\sqrt{\Delta \omega^{2}\left(\Omega_{z}^{2}+\omega^{2}\right)+2 \Omega_{z}^{2} \omega^{2}}}, \\
\lambda_{2}=\sqrt{\Omega_{z}^{2}+\omega^{2}+\Delta \omega^{2} / 4-\sqrt{\Delta \omega^{2}\left(\Omega_{z}^{2}+\omega^{2}\right)+2 \Omega_{z}^{2} \omega^{2}}} .
\end{array}\right.
$$

For the case of an initially mode-matched gyroscope ( $\Delta \omega=0$ for $\Omega_{z}=0$ ), the solution (2) simplifies to:

$$
\left\{\begin{array}{l}
\lambda_{1}=\omega+\Omega_{z}, \\
\lambda_{2}=\omega-\Omega_{z} .
\end{array}\right.
$$

These expressions for the modal frequencies in presence of rotation reveal that the mechanical modal frequencies $\lambda_{I, 2}$ of the gyroscope structure are modulated by the input angular rate $\Omega_{z}$, Figure 2. This rate-dependent change in modal frequencies presents a challenge for high- $Q$ mode-matched gyroscopes (rotation yields in-operation mode-detuning, which limits the useful linear range).

\section{Frequency-Based Detection of Input Rate}

In this paper, we propose to take advantage of the modal frequency splitting to enable direct FM measurement of the input rotation rate. Based on the eigen-frequency solution (3) for an initially mode-matched gyroscope, the input rotation rate $\Omega_{z}$ can be measured from the observed modal frequencies $\lambda_{1,2}$ according to a linear expression:

$$
\Omega_{z}=\frac{1}{2}\left(\lambda_{1}-\lambda_{2}\right) \text { for } \Delta \omega=0 .
$$
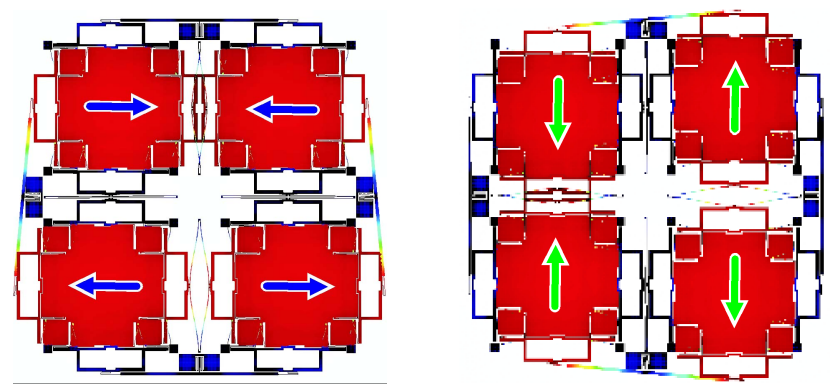

Figure 4: QMG provides balanced, low dissipation modes of $x$ - and $y$-axis vibrations at the same frequency, FEM.

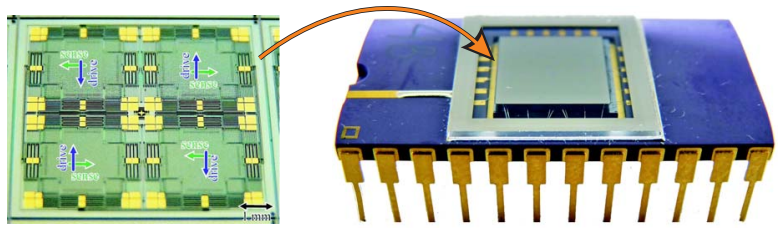

Figure 5: Photograph of an SOI prototype of $Q M G$ vacuum packaged at sub-mTorr cavity pressure $w /$ getters.

The investigated FM based operational principle can also be extended to the more general case of frequency mismatched vibratory rate gyroscope $(\Delta \omega>0)$ by solving the system of equations (2) for the input angular rate $\Omega_{z}$ :

$$
\Omega_{z}=\frac{1}{2} \sqrt{\left(\lambda_{1}-\lambda_{2}\right)^{2}-\frac{4 \omega^{2} \Delta \omega^{2}}{4 \omega^{2}+\Delta \omega^{2}-\left(\lambda_{1}-\lambda_{2}\right)^{2}}} .
$$

The obtained closed-form solutions (4)-(5) provide the basis for the frequency based measurement of the input rate by tracking the modal frequency split in a high- $Q$, nearly mode-matched vibratory gyroscope. The quasi-digital FM approach resolves the $Q$ versus linear range and bandwidth tradeoff of conventional analogue AM based vibratory rate sensors.

Maximization of the FM-operated gyroscope $Q$ factor improves the frequency stability and rate resolution. At the same time, the linear input range of an FM operated gyroscope is independent of the $Q$ and is only limited by the device nominal frequency (designing a gyroscope to operate above $1 \mathrm{kHz}$ guaranties a linear range $>72,000 \mathrm{deg} / \mathrm{s}$ ). The modal frequency split provides an instantaneous measure of the input rate, allowing the use of ultra-high $Q$ structures without the sensor bandwidth sacrifice. An additional advantage of the approach is the self-calibration against common drifts in the modal frequencies enabled by the differential frequency measurement.

\section{HIGH Q TRANSDUCER DESIGN}

In this section we describe a symmetric, ultra-high $Q$ vibratory gyroscope structure tailored for FM operation.

\section{Design Requirements}

Several design criteria must be met by the mechanical sensor element to fully realize the advantages of the proposed FM operation. A geometrically mode-matched structure is needed to optimize the minimal detectable 


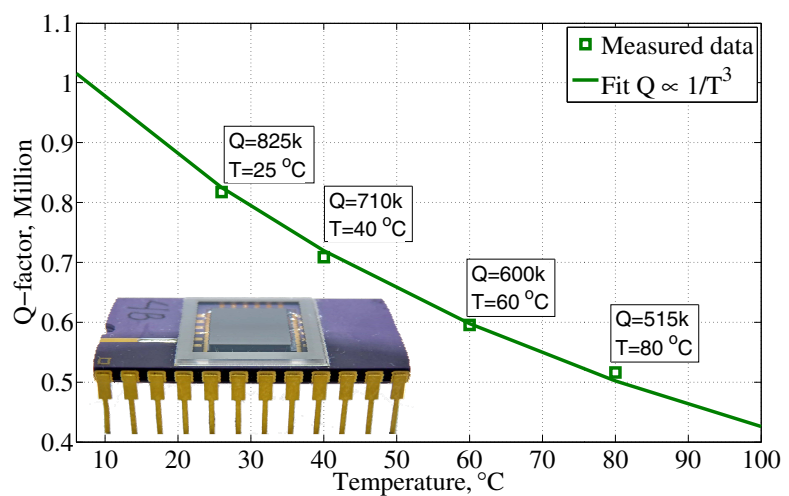

Figure 6: Structural and temperature characterization of a vacuum sealed $Q M G$ reveals isotropic $Q$ close to 1 Million.

angular rate signal and increase temperature stability of the sensor. At the same time, identical ultra-high Q is needed in both modes of mechanical vibration to maximize the frequency stability and rate resolution. These requirements are satisfied by an $x-y$ symmetric, dynamically balanced, anti-phase operated gyroscope, such as the recently introduced Quadruple Mass Gyroscope (QMG) [6].

\section{Quadruple Mass Gyroscope Architecture}

The mechanical structure of the QMG mechanical sensor element comprises four identical symmetrically decoupled tines with linear coupling flexures as well as a pair of anti-phase synchronization lever mechanisms for both the $x$ - and the $y$-mode, Figure 3. This $x-y$ symmetric system of four anti-phase tines provides the structure with two anti-phase, dynamically balanced modes of mechanical vibration at a single operational frequency, Figure 4. The complete $x-y$ structural symmetry of the device improves robustness of frequency matching against fabrication imperfections and temperature induced frequency drifts. An additional advantage of the QMG anti-phase levered architecture is the mechanical suppression of common mode displacement in the coupled tines.

\section{EXPERIMENTAL CHARACTERIZATION}

In this section we experimentally evaluate the FM based rate detection approach using a vacuum sealed QMG.

\section{Prototype Fabrication and Packaging}

The QMG sensor element used for the experimental characterization was fabricated using an in-house, single mask, wafer scale SOI process with a conductive $100 \mu \mathrm{m}$ thick device layer and a $5 \mu \mathrm{m}$ thick buried oxide. First, a hard mask for sensor structures was defined by patterning a thermally grown $1 \mu \mathrm{m}$ layer of surface oxide with a Surface Technology Systems (STS) Advanced Oxide Etching (ASE) tool. The gyroscope structures were then defined by DRIE using a Unaxis Versaline VL-7339 tool. The singulated sensors were released using a timed $20 \%$ hydrofluoric acid wet etch. The released QMG devices were bonded to ceramic DIP packages using Au-Sn eutectic solder and wire bonded. To enable stand-alone ultra-high $Q$ operation, the

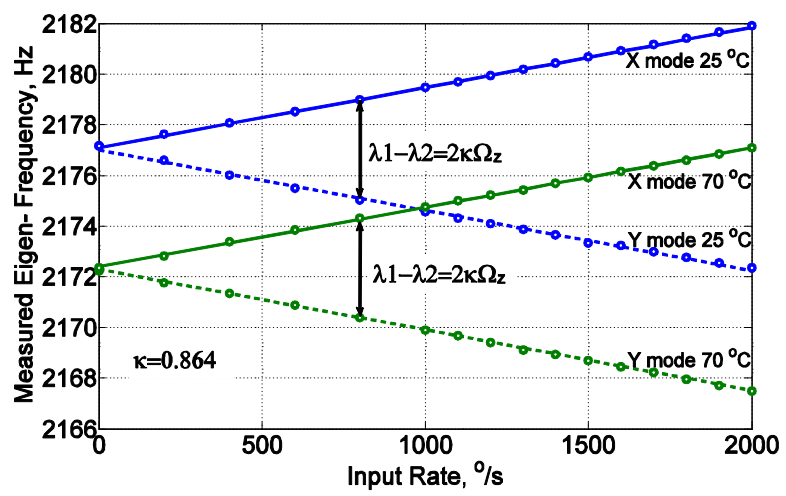

(a) Differential FM detection of the $\Omega_{z}$ input rate from the modal frequency split $\left(\lambda_{1}-\lambda_{2}\right)$ is invariant to temperature.

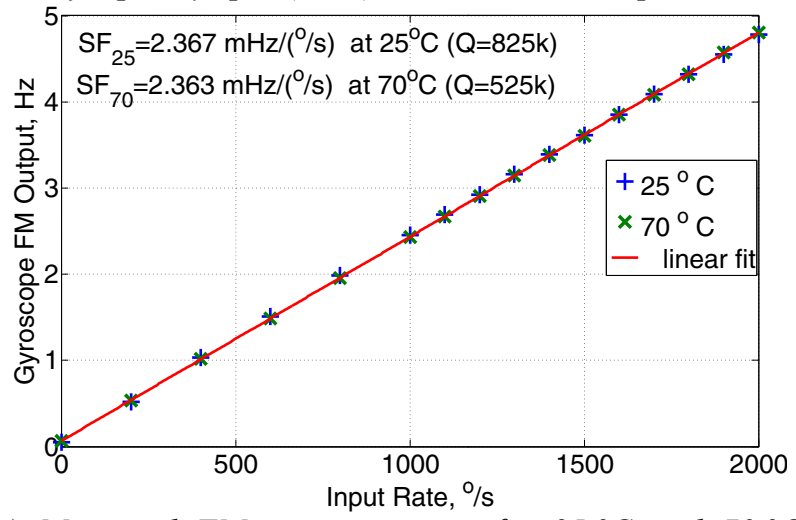

(b) Measured FM rate responses for $25^{\circ} \mathrm{C}$ and $70^{\circ} \mathrm{C}$ using differential detection of the modal frequency split.

Figure 7: Characterization of the FM-based rate system reveals less than $0.2 \%$ response drift between $25^{\circ} \mathrm{C}$ and $70{ }^{\circ} \mathrm{C}$ (without any temperature compensation) despite a $40 \% \mathrm{Q}$ reduction and a $5 \mathrm{~Hz}$ drop of nominal frequency.

packaged sensors were vacuum sealed using custom made glass lids with getter material [7] providing robust submTorr vacuum inside the package cavities, Figure 5.

\section{Structural Characterization}

The dynamically balanced anti-phase design and vacuum packaging of QMG sensors was expected to yield isotropic, ultra-high $Q$-factors for stable FM operation. The structural $x$ - and $y$-modes a sealed QMG prototype with a nominal frequency of $2.2 \mathrm{kHz}$ were experimentally characterized at different temperatures using ring-down tests. Exponential fits of the time domain amplitude decay data revealed a room temperature $Q$-factor of 0.83 million and demonstrated Q-factor in excess of 0.5 million up to $80^{\circ} \mathrm{C}$, Figure 6 .

\section{Rate Characterization}

In conventional AM-operated vibratory rate gyroscopes, drifts of modal frequencies and $Q$-factors are major sources of sensor output scale factor and bias drift over temperature. Theoretical analysis of the proposed FM rate sensor suggest immunity against these drift mechanisms 


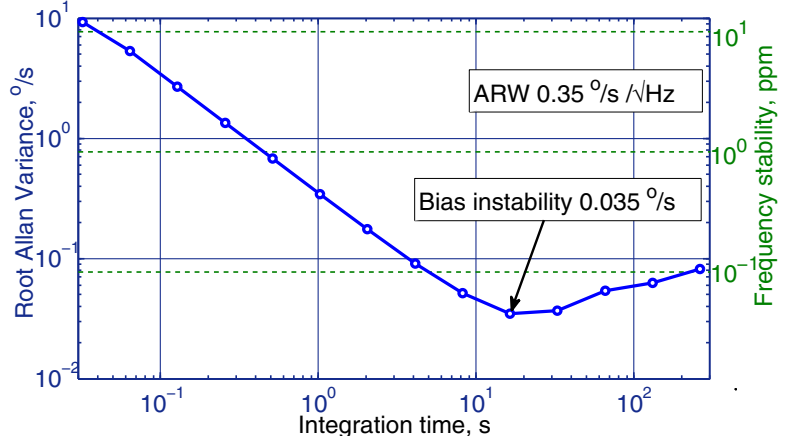

Figure 8: Measured Allan variance of the FM sensor showing $0.35 \mathrm{deg} / \mathrm{s} / \mathrm{V} \mathrm{Hz}$ resolutions and $0.035 \mathrm{deg} / \mathrm{s}$ bias.

by virtue of the differential frequency detection. To experimentally investigate this hypothesis, a vacuum packaged QMG sensor instrumented for real-time FM operation was characterized on a temperature controlled Ideal Aerosmith $1291 \mathrm{BR}$ rate table at $25^{\circ} \mathrm{C}$ and $70^{\circ} \mathrm{C}$.

For these experiments, the $x$ - and $y$-modes of the QMG device were electrostatically excited into resonances using a combination of $0.1 \mathrm{Vdc}$ bias and $0.1 \mathrm{Vac}$ driving signals produced by two separate digital PLLs. The motional signals for both modes of vibration were detected using capacitive detection with electromechanical amplitude modulation (EAM). The two modal frequencies of the gyroscope mechanical structure were continuously monitored by the two PLLs, Figure 7.

As theoretically expected for a mode-matched gyroscope, the measured split between the nominally equal modal frequencies was directly proportional to the input rate, Figure 7(a). The linear input-output characteristic of the FM instrumented QMG with $Q$-factor on the order of 1 million was confirmed up to the $2000 \mathrm{deg} / \mathrm{s}$ rotation rate limit of the rate table. Unlike the conventional AM approach, the FM detection of the input rate from the modal frequency split demonstrated invariance to changes in temperature and $Q$-factors, Figure $6(\mathrm{~b})$. Without any active temperature compensation, experimental characterization of the $\mathrm{FM}$ angular rate sensor at $25^{\circ} \mathrm{C}$ and $70{ }^{\circ} \mathrm{C}$ revealed less than $0.2 \%$ response fluctuation (within the accuracy of the experimental setup) despite a $40 \%$ reduction of the $Q$-factor and a $5 \mathrm{~Hz}$ drop of the nominal frequency.

Noise performance of the FM angular rate system is driven by the frequency stability of the two modes of vibration in the QMG structure. Preliminary noise characterization of the FM-instrumented QMG was performed by measuring the modal frequency stability at zero input rate in room temperature. The frequency data was converted to equivalent rate using the measured scale factor of $2.36 \mathrm{mHz} /(\mathrm{deg} / \mathrm{s})$, Figure 6 . In this experiment, the measured $0.05 \mathrm{ppm}$ instability of frequency translates into $0.035 \mathrm{deg} / \mathrm{s}$ rate bias. The sensor resolution can be further improved by implementing an automatic gain control, which reduces the phase noise and helps stabilize frequency [8].

\section{CONCLUSION}

We demonstrated a quasi-digital angular rate sensor based on mechanical FM. This novel approach eliminates the gain-bandwidth tradeoff of conventional AM gyroscopes and may enable signal-to-noise ratio improvements through the use of ultra-high $Q$-factor structures without limiting the measurement bandwidth and range. A differential frequency measurement enables simultaneous detection and decoupling of the input angular rate and the device temperature. In other words, the gyroscope becomes its own thermometer, eliminating thermal lags and hysteresis issues.

The novel approach was experimentally characterized using an in-house developed, vacuum packaged, dynamically balanced Quadruple Mass Gyroscope (QMG) with a $2.2 \mathrm{kHz}$ operational frequency and a measured $Q$ factor of 0.8 million. Advantages of the FM-instrumented QMG over the current state-of-the-art AM based gyroscopes include extremely wide linear and dynamic ranges $(>72,000$ $\mathrm{deg} / \mathrm{s}$ and $>110 \mathrm{~dB}$, respectively), wide bandwidth $(>100$ $\mathrm{Hz}$ ), immunity to temperature variations, and robustness to external mechanical and electromagnetic interferences.

\section{ACKNOWLEDGMENT}

This work was supported by the ONR/NSWDD grant N00178-08-C1014. The authors acknowledge H. Florence of SAES Getters and P. Barnes of SST International for help with vacuum sealing. Devices were designed and characterized at the UC Irvine MicroSystems Laboratory.

\section{REFERENCES}

[1] M. Weinberg et al., "Energy Loss in MEMS Resonators and the Impact on Inertial and RF Devices", Proc. TRANSDUCERS 2009, pp. 688-695, 2009.

[2] M.F. Zaman et al., "A Mode-Matched Silicon-Yaw Tuning-Fork Gyroscope with Subdegree-Per-Hour Allan Deviation Bias Instability," IEEE JMEMS, vol. 17, no. 6, pp. 1526-1536, 2008.

[3] T. R. Albrecht et al., "Frequency Modulation Detection Using High-Q Cantilevers for Enhanced Force Microscope Sensitivity," J. Appl. Phys. 69, pp. 668$673,1991$.

[4] A. A. Seshia et al., "An Integrated Microelectromechanical Resonant-Output Gyroscope," IEEE MEMS 2002, Las Vegas, NV, pp. 722-726, 2002.

[5] C. Comi et al.,"A High Sensitivity Uniaxial Resonant Accelerometer," Proc. IEEE MEMS 2010, pp. 260-263.

[6] A.A. Trusov et al., "Ultra-High Q Silicon Gyroscopes with Interchangeable Rate and Whole Angle Modes of Operation," Proc. IEEE Sensors 2010, pp. 864-867.

[7] A.R. Schofield et al. "Versatile Sub-mTorr Vacuum Packaging for the Experimental Study of Resonant MEMS," Proc. IEEE MEMS 2010, pp. 516-519, 2010.

[8] L. Seungbae, C.T.-C. Nguyen, "Influence of Automatic Level Control on Micromechanical Resonator Oscillator Phase Noise," IEEE International Frequency Control Symposium, vol. 22, no. 1, pp. 341-349, 2003. 